\title{
A Classification of Dialogue Actions in Tutorial Dialogue
}

\author{
Mark Buckley and Magdalena Wolska \\ Dept. of Computational Linguistics \\ Saarland University \\ 66041 Saarbrücken, Germany \\ \{buckley|magda\}@coli.uni-sb.de
}

\begin{abstract}
In this paper we present a taxonomy of dialogue moves which describe the actions that students and tutors perform in tutorial dialogue. We are motivated by the need for a categorisation of such actions in order to develop computational models for tutorial dialogue. As such, we build both on existing work on dialogue move categorisation for tutorial dialogue as well as dialogue taxonomies for general dialogue. Our taxonomy has been prepared by analysing a corpus of tutorial dialogues on mathematical theorem proving. We also detail an annotation experiment in which we apply the taxonomy and discuss idiosyncrasies in the data which influence the decisions in the dialogue move classification.
\end{abstract}

\section{Introduction}

The field of Intelligent Tutoring Systems has seen recent developments moving towards adding natural language capabilities to computer-based tutoring (Graesser et al., 1999; Zinn, 2004; Litman and Silliman, 2004), motivated by empirical investigations which point to the effectiveness of human tutors (Bloom, 1984; Moore, 1993; Graesser et al., 1995). However, to be able to interact with a student through the medium of natural language dialogue, the system must have a model of how such tutorial dialogues can progress and what utterances are licenced. In order to develop such a model of dialogue, we need to understand and describe the "actions" performed with words, i.e. speech acts (Austin, 1955) or dialogue moves. This involves identifying and categorising the functions

(c) 2008. Licensed under the Creative Commons Attribution-Noncommercial-Share Alike 3.0 Unported license (http://creativecommons.org/licenses/by-nc-sa/3.0/). Some rights reserved. that utterances may have in dialogue and their relationships to each other.

Researchers in conversation and dialogue theory have proposed various general categorisations of dialogue moves. DIT++ (Bunt, 2000) is an example of a comprehensive multidimensional taxonomy of dialogue acts for information dialogues based on DAMSL (Allen and Core, 1997), a general-purpose extensible taxonomy proposed as a standard for dialogue annotation. The DAMSL dialogue act taxonomy characterises utterances along four dimensions which correspond to four levels of functions utterances may have. The forward looking function describes the utterance's effect on the following interaction, the backward looking function, its relation to previous dialogue, the communicative status describes the comprehensibility or interpretability of the utterance, and the information level characterises the content of the utterance.

Tsovaltzi and Karagjosova (2004) proposed an extension of the DAMSL classification based on an analysis of tutorial dialogue corpora. The proposed taxonomy adds a Task dimension which concentrates on tutor actions in the dialogue. ${ }^{1}$ Building on this work, we propose a further extension to this taxonomy inspired by our analysis from the point of view of task-related goals. The classification we present (i) includes modifications of the DAMSL categorisation motivated by tutorial dialogue, (ii) accounts for student's actions, and (iii) introduces a Task progress dimension whose purpose is to characterise the completion status of a generally viewed "task", instantiated for the purpose of tutorial dialogue. We validated our dialogue act categorisation in a small-scale annotation experiment whose results we present.

This paper is organised as follows: In Section 2 we introduce our data and taxonomy development

\footnotetext{
${ }^{1}$ This classification has not been, to our knowledge, quantitatively evaluated.
} 
methodology. In Section 3 we present the taxonomy. The results of the annotation experiment and their discussion are presented in Section 4. Section 5 presents related work and Section 6 concludes the paper.

\section{The Data and Analysis Methodology}

Our work is based on an analysis of two corpora of tutorial dialogues on mathematical theorem proving. We use the data to (i) verify the general dialogue dimensions of the DAMSL taxonomy in the context of tutorial dialogues, and (ii) extend the taxonomy by developing the task dimension. While the specific task-level moves are instantiated for the mathematics tutoring domain, our aim is to maintain generality that would allow us to model task-level moves in other tutoring domains as well as task-oriented dialogues in general. Below we briefly introduce the corpora and outline our methodology.

Corpora We analysed two corpora of tutorial dialogues in the domain of mathematical theorem proving collected in Wizard-of-Oz experiments in which a human tutor (wizard) simulated the system's behaviour (Benzmüller et al., 2003; Benzmüller et al., 2006a). The domains of mathematics in the first (Corpus-I) and second (CorpusII) corpora are naive set theory and binary relations respectively (Wolska et al., 2004; Benzmüller et al., 2006b).

In both experiments the dialogues were conducted in German using the keyboard and a graphical user interface. The tutoring in the experiment described in (Benzmüller et al., 2003) was performed under three experimental conditions. The control group ( 8 subjects) were tutored according to the minimal feedback strategy in which the tutor's reactions were limited to informing the student as to the correctness and completeness of their contributions. In the didactic group (7 subjects) and socratic group (7 subjects) the tutor's strategy focused on disclosing partial solutions to the student in case of lack of progress and leading toward the solution (hinting) respectively. Given the tutoring strategy the verbosity of the minimal feedback tutors was limited, while in both other conditions as well as in the other experiment, the subjects and the tutors were unconstrained in terms of the linguistic realisation of their turns. Table 1 shows the overview of the size of the corpora in terms of the number of turns.

\begin{tabular}{lll}
\hline & Corpus-I & Corpus-II \\
\hline no. subjects & 22 & 37 \\
no. turns & 775 & 1917 \\
no. student turns & 332 & 937 \\
no. tutor turns & 443 & 980 \\
\hline
\end{tabular}

Table 1: Overview of the corpora

Methodology In developing the taxonomy we pursued the following iterative methodology:

First, in order to build the initial taxonomy, we analysed 18 dialogues from Corpus-I containing 299 utterances (the development set). The purpose of this analysis was to (i) verify the general suitability of the DAMSL scheme in the tutoring domain, ${ }^{2}$ (ii) identify features of dialogues moves relevant in tutoring that were not present in the original taxonomy (see the discussion in Section 4), (iii) identify an initial set of task-level moves. We descriptively defined the move types and wrote draft annotation guidelines.

Second, we applied the initial taxonomy to 4 dialogues (108 utterances) taken from the two corpora in an annotation task performed independently by the authors of this paper (a preliminary test set), after which we extended the taxonomy and refined the existing category definitions. Finally, we randomly ${ }^{3}$ selected an 64-utterance subset taken from both corpora (validation set) to test the coverage of the final taxonomy.

\section{A Dialogue Move Taxonomy}

Our goal in the analysis of the development set and preliminary test set of the corpus was to determine a categorisation of the actions that can be performed by students and tutors in tutorial dialogues. The taxonomy which we have created from this categorisation contains the dialogue moves which realise these actions. Now the utterances performed by students and tutors realise actions which may or may not address or have an effect on the current task. We thus speak of the task-level function of an utterance in addition to a general dialogue level function which all utterances have. Task-level vs. general dialogue level function is therefore the basic split in our taxonomy.

\footnotetext{
${ }^{2}$ We expected this to be suitable because DAMSL is a taxonomy with general applicability.

${ }^{3}$ We avoided dialogues in which the student's utterances contained formulas only.
} 
The full definition of the taxonomy is presented in Table 2. For each dialogue move we give a short explanation of what function it is intended to capture as well as a short example. In the following sections we discuss some of our design decisions.

\subsection{The Forward and Backward Dimensions}

At the general dialogue level we follow the DAMSL taxonomy and categorise the functions of utterances according to their relationship with the previous dialogue and their effect on the dialogue to follow. For these functions we use a Forward dimension and a Backward dimension, respectively. In general, we try to accommodate the DAMSL categories in order to build as much as possible on existing generally accepted work on dialogue moves. The forward dimension captures utterances which are either assertions, requests or commands. The backward dimension captures utterances which agree or disagree with previous utterances, address questions, signal the understanding status of previous utterances, or stand in some information relation to previous utterances. The main differences between DAMSL and our categorisation within these two dimensions are the following: (i) we combine DAMSL's Assert and Re-assert in a single category Assert which may be optionally marked as repeating information, (ii) we combine DAMSL's Action-directive and Info-request in a higher-level category of Requests, (iii) in place of DAMSL's Answer, we introduce a more general Address category in the backward dimension with subcategories Answer, Deflect, and Neutral, where Deflect accounts for avoiding answering and Neutral refers to those utterances which simply address a previous information request without answering it or a previous action directive without acceding to it. The remaining DAMSL categories were left unchanged; they are also not presented in Table 2.

\subsection{The Task and Task Progress Dimensions}

At the task level we have utterances which address the task at hand. These can mean altering the state of the task solution, for instance by performing a step in the solution, or talking about the task solution without altering it, for instance making statements about previously performed steps. We divide the task related actions in those which address the task directly and those which address the solution construction process, and capture these in the task and task progress dimensions respectively.
The Task dimension contains most of the task related dialogue moves. We follow Tsovaltzi and Karagjosova (2004) by splitting the task dimension into two subdivisions which relate to the parallel tasks being carried out and the roles of the dialogue participants. Since the roles of student and tutor restrict what actions can be performed by the speakers, we split the task dimension into actions which contribute to the solving task and those which contribute to the teaching task. Actions in the solving task are typically performed by the student, actions in the teaching task are typically performed by the tutor and are pedagogically motivated. This is important for example to differentiate between requests for task level information - requests coming from the student are of an information seeking nature, those that come from the tutor contribute to diagnostic or pedagogical goals.

Within the solving task, changes or additions to the solution are captured by Solution-step, which may be a new step or an extension of an existing step, and Solution-strategy. Solution-strategy is divided into stating a strategy which will be followed and stating a solution step which will be performed in the future. The difference between these is that the statement of a future step refers to a single step in the solution which the student is committing to perform, whereas a strategy is a more abstract concept. A strategy is more like a solution approach which may consist of a number of steps, however which actual steps are to be performed is left open. In the domain of mathematical theorem proving a strategy may refer to a particular proving technique, for instance a proof by induction or by contradiction, which may be realised by an utterance such as "I will now do a proof by induction". Exactly what constitutes a step and a strategy is a matter of how the domain is modelled.

Request-assistance covers actions which ask for help with the task. Within the teaching task, Solution-step-evaluation refers to utterances that convey the correctness or otherwise of steps. Domain relevant requests ask for domain related information such as definitions. Hint covers direct hints about how to continue the task, for instance by giving away a concept which should be used by the student. The hint category will need to cover many different kinds of actions depending on the tutorial strategy which tutors follow - we have not subdivided this category and refer to Tsovaltzi et al. (2004) where this is further elaborated. 
The Task Progress dimension equates in part to the task management dimension in DAMSL. Here the dialogue moves related to the current task are those which start, finish, restart or abandon it. The student can indicate the status of the solution construction process to be on-track or finished or signal that he is lost.

In summary, we have prepared our taxonomy of dialogue moves for tutoring by adding a Task and Task Progress dimensions to the original DAMSL taxonomy. We have tried to keep as close to the DAMSL specification as possible with regard to the general dialogue level function of dialogue moves, while at the same time adapting it to capture the phenomena of tutorial dialogue. Although some moves will typically by performed by either the student or the tutor (for example, only the tutor will realistically give hints) we do not introduce any constraints which restrict this.

\section{Validating the Taxonomy}

We used the taxonomy to perform a small-scale annotation experiment on a validation set taken from the two corpora introduced in Section 2. The data had previously been segmented into utterances. The goal of this experiment was to see whether our categorisation can be reliably applied to data and to validate the coverage of the taxonomy. The annotation was carried out by two annotators (the authors of this paper), following the definitions of the dialogue moves informally presented above. We did not consider the category information-relation because no definition is given by the original DAMSL taxonomy, however we will return to the question of information relation later in the discussion.

Results Inter-annotator agreement was calculated using Cohen's kappa (Cohen, 1960) and the results of the experiment are given in the following table.

\begin{tabular}{|l|l|}
\hline Dimension & $\kappa$ value \\
\hline Forward & 0.87 \\
Backward & 0.47 \\
Task & 0.75 \\
Task Progress & 0.91 \\
\hline
\end{tabular}

These results can be considered very good for the Forward and Task Progress dimensions, good for the Task dimension, and low for the Backward dimension. Among the categories with the lowest agreement were Neutral at 0.11 and Stepaugmentation at 0.37 . In this preliminary evaluation our strategy was not to use an category "other" for utterances which did not appear to belong to any existing category, but rather to try to fit the annotation to the categories as they are. We marked possibly problematic utterances for further discussion.

Example We give examples of two fully annotated dialogue excerpts in Figure 1. The examples illustrate some of the types of problematic utterances which the corpora contain. For instance both utterances "Really?" and "Yes?" are questions and could appear to be information requests, but in fact act more like prompts to continue, for which we had no category. Similarly the functions of the questions in sequence in the second example are difficult to abstract. We have tagged these as Neutral, since they discharge the obligations introduced by the questions before them, but the link between consecutive interrogative utterances is elusive.

Discussion We will now briefly discuss the results and findings of our annotation experiment and allude to some of the possible causes of the difficulties we encountered.

The nature of tutorial dialogue is an underlying factor which makes it difficult to annotate categories reliably. Students tend to be very concise, which makes it difficult to determine how the student intended to relate the latest input to the previous discourse. This is reflected in our agreement score for the backward dimension, which at 0.47 is much lower than the other dimensions, as well as in the agreement score of 0.37 for the Solutionstep augmentation category, which is heavily dependent on previous context. This result may even point to a general characteristic of tutorial dialogue which makes computational modelling challenging. In particular the Neutral category resulted in conflicting annotations because it is often unclear, as in the examples shown above, whether Requests are being answered or merely addressed.

We have found that tutors typically perform utterances which contribute to many different goals - for instance they can simultaneously reject proposed solution steps while giving hints on how to continue in the task. The purpose of multidimensional dialogue move taxonomies is to handle this very multifunctionality, and while this is success- 


\begin{tabular}{|c|c|c|c|}
\hline Utterance & Forward & Backward & Task \\
\hline $\begin{array}{l}\text { S: It holds that } P(C \cup(A \cap B)) \subseteq P(C) \cup \ldots \\
\text { T: Really? } \\
\text { S: no it's not, } \\
\text { S: the other way around } \\
\text { T: that's right at last }\end{array}$ & $\begin{array}{l}\text { assert } \\
\text { info-request } \\
\text { assert } \\
\text { assert }\end{array}$ & $\begin{array}{l}\text { reject } \\
\text { answer } \\
\text { accept }\end{array}$ & $\begin{array}{l}\text { solution-step:new } \\
\text { signal-incorrect } \\
\text { solution-step:new } \\
\text { signal-correct }\end{array}$ \\
\hline $\begin{array}{l}\mathrm{S}: \mathrm{R} \circ \mathrm{S}:=\{(\mathrm{x}, \mathrm{y}) \mid \exists \mathrm{z}(\mathrm{z} \in \mathrm{M} \wedge(\mathrm{x}, \mathrm{z}) \in \mathrm{R} \wedge \ldots \\
\mathrm{T}: \text { That's right! } \\
\mathrm{S}: \text { now i want the inverse of that } \\
\mathrm{T}: \text { yes? } \\
\mathrm{S}:(\mathrm{R} \circ \mathrm{S})^{-1} \\
\mathrm{~T}:=\text { ? } \\
\mathrm{S}: \text { How will the system answer? } \\
\mathrm{T}: \text { What's the question? } \\
\mathrm{S}: \text { Can the system conclude }(\mathrm{R} \circ \mathrm{S})^{-1} \text { from } \mathrm{R} \circ \mathrm{S} \\
\mathrm{T}: \text { yes } \\
\mathrm{T}: \text { But try it yourself! }\end{array}$ & $\begin{array}{l}\text { assert } \\
\text { assert } \\
\text { assert } \\
\text { assert } \\
\text { info-request } \\
\text { info-request } \\
\text { info-request } \\
\text { info-request } \\
\text { assert } \\
\text { action-dir }\end{array}$ & $\begin{array}{l}\text { accept } \\
\text { neutral } \\
\text { neutral } \\
\text { request-clar } \\
\text { neutral } \\
\text { neutral } \\
\text { neutral } \\
\text { answer }\end{array}$ & $\begin{array}{l}\text { solution-step:new } \\
\text { signal-correct } \\
\text { state-future-step } \\
\text { hint } \\
\text { solution-step:new } \\
\text { request-explanation }\end{array}$ \\
\hline
\end{tabular}

Figure 1: Annotated example from the corpus

ful to a point, conflicts in the annotation experiment have highlighted some dual functions within the same category. For instance, utterances simultaneously rejecting steps and requesting explanations of the errors in the steps were found a number of times.

We have found at least three categories that may need to be added to the current taxonomy to make it cover tutorial dialogue more completely. As discussed above, a prompt type in the forward dimension seems necessary. In addition, we would foresee a backward category which corrects a previous utterance, a category in the solving task which requests the next step in the solution, and a category in the task progress dimension to check if the current task is being restarted. Similar categories are proposed by Tsovaltzi and Karagjosova (2004), and may be taken up.

We can draw attention to the fact that there are many interrelations between the dimensions which are not captured by our presentation of the taxonomy, and which may for instance be accounted for by introducing constraints on label combinations. We observe that many utterances stand in some information relation (a DAMSL category) to the previous discourse, although we have not further specified what this relation might be. Such utterances are typically step augmentations, and could be described for instance (in RST terms) as elaborations.

Finally we have adopted Tsovaltzi and Karagjosova's top-level structure, with Task as a dimension. However, we observe that it would be equally valid and more in keeping with the original DAMSL categorisation of utterance functions to make use of the existing Task sub-category of the Info-level dimension. Similarly, our Task progress corresponds to Info-level's sub-category Task management. This is a straightforward structural change which will not affect the annotation results within these categories.

\section{Related Work}

The original DAMSL taxonomy was applied to and evaluated on the TRAINS corpus of problem solving dialogues (Core and Allen, 1997). In this annotation a single label Task was used to mark all task-related utterances. In the Verbmobil project, a set of dialogue moves specific to negotiation was proposed (Alexandersson et al., 1997). These moves capture only the task-specific functions of the utterances. Similarly, the HCRC Map Task coding scheme concentrates on the task functions of utterances, here specific to instruction-giving dialogues. This classification is based on conversational games approach to dialogue semantics (Houghton, 1986).

The DIT++ taxonomy of dialogue acts (Bunt, 2006) provides a more fine grained categorisation of general dialogue actions, however there is no one category or dimension dedicated to task specific functions. The category closest to (a subset of) our Task dimension would be Activity-Specific Functions, which however is defined in terms of performative verbs or graphical actions. In the tutoring domain not all task-related actions are realised by performatives.

There are a number of categorisations of dialogue actions specific to tutoring and motivated by the development of tutorial dialogue systems. Closely related to our work is a recent study by 
Porayska-Pomsta et al. (2008), who categorise task related student actions and tutor feedback in a investigation of student affect. (Dzikovska et al., 2006) propose a flat coding scheme for tutorial dialogues on mathematics and relate it to a model of collaborative problem solving dialogue. A simpler taxonomy is presented by Marineau et al. (2000) which differs from our approach in that it was developed with the goal of automatic classification in an intelligent tutoring system. In a pedagogically motivated analysis of a corpus of tutorial dialogues on computer literacy, Graesser et al. (1999) categorise tutors' actions in order to propose a model of tutorial dialogue structure.

\section{Conclusions and Future Work}

In this paper we have presented a taxonomy of dialogue moves which captures the actions performed in by students and tutors in a corpus of tutorial dialogues. We then detailed an annotation experiment which applied the taxonomy to a validation data set and achieved good inter-annotator agreement. This preliminary study showed that we are able to cover the data well. We did however find a number of problematic phenomena in the data, such as that of relating task level actions to the previous discourse, which are of particular importance for classifying tutorial dialogue actions.

In our future work we plan a larger scale annotation of a further test set of our corpus, which we believe will confirm the tendencies found so far. We also intend to apply our taxonomy in an annotation of tutorial dialogue dealing with different task domains, for example, tutorial dialogue corpora in domains other than mathematics and general problem-solving dialogues (e.g. TRAINS). One of the goals of our work is to inform the development of models for tutorial dialogue, and so with a view towards operationalisation of the dialogue moves in our taxonomy, we will work on an axiomatic formalisation of the dialogue moves. This can form important input into developing a plan-based model for tutorial dialogue.

\section{References}

Alexandersson, Jan, Bianka Buschbeck-Wolf, Tsutomu Fujinami, Elisabeth Maier, Norbert Reithinger, Birte Schmitz, and Melanie Siegel. 1997. Dialogue acts in verbmobil-2. Technical report, DFKI.

Allen, James and Mark Core. 1997. Draft of DAMSL: Dialogue act markup in several layers.
DRI: Discourse Research Initiative, University of Pennsylvania. http://www.cs.rochester. edu/research/cisd/resources/damsl/ RevisedManual/.

Austin, John L. 1955. How to do things with Words. 2005, second edition. William James Lectures.

Benzmüller, Christoph, Armin Fiedler, Malte Gabsdil, Helmut Horacek, Ivana Kruijff-Korbayová, Manfred Pinkal, Jörg Siekmann, Dimitra Tsovaltzi, Bao Quoc Vo, and Magdalena Wolska. 2003. A Wizard-of$\mathrm{Oz}$ experiment for tutorial dialogues in mathematics. In Aleven, Vincent, Ulrich Hoppe, Judy Kay, Riichiro Mizoguchi, Helen Pain, Felisa Verdejo, and Kalina Yacef, editors, AIED2003 Supplementary Proceedings, volume VIII: Advanced Technologies for Mathematics Education, pages 471-481, Sydney, Australia. School of Information Technologies, University of Sydney.

Benzmüller, Christoph, Helmut Horacek, Ivana KruijffKorbayová, Henri Lesourd, Marvin Schiller, and Magdalena Wolska. 2006a. DiaWozII - A Tool for Wizard-of-Oz Experiments in Mathematics. In Proceedings of the 29th Annual German Conference on Artificial Intelligence (KI-06), Lecture Notes in Computer Science, number 4314, pages 159-173, Bremen, Germany. Springer-Verlag.

Benzmüller, Christoph, Helmut Horacek, Henri Lesourd, Ivana Kruijff-Korbayová, Marvin Schiller, and Magdalena Wolska. 2006b. A corpus of tutorial dialogs on theorem proving; the influence of the presentation of the study-material. In Proceedings of the 5th International Conference on Language Resources and Evaluation (LREC-06), pages 17661769, Genoa, Italy. ELDA.

Bloom, B. 1984. The 2 Sigma Problem: The Search for Methods of Group Instruction as Effective as One-toOne Tutoring. Educational Researcher, 13(6):4-16.

Bunt, Harry. 2000. Dialogue pragmatics and context specification. In Bunt, Harry and William Black, editors, Abduction, Belief and Context in Dialogue. Studies in Computational Pragmatics, volume 1, pages $81-150$. Benjamins.

Bunt, Harry. 2006. Dimensions in Dialogue Act Annotation. In Proceedings of the 5th International Conference on Language Resources and Evaluation (LREC-06), pages 919-924, Genova, Italy.

Cohen, Jacob. 1960. A coefficient of agreement for nominal scales. Educational and Psychological Measurement, 20(1):37-46.

Core, Mark G. and James F. Allen. 1997. Coding dialogues with the DAMSL annotation scheme. In Traum, David, editor, Working Notes: AAAI Fall Symposium on Communicative Action in Humans and Machines, pages 28-35. AAAI, Menlo Park, CA, USA. 
Dzikovska, Myroslava O., Charles B. Callaway, Matthew Stone, and Johanna D. Moore. 2006. Understanding student input for tutorial dialogue in procedural domains. In Schlangen, David and Raquel Fernandez, editors, Proceedings of Brandial, the 10th Workshop on the Semantics and Pragmatics of Dialogue, pages 10-17.

Graesser, A. C., N. K. Person, and J. P. Magliano. 1995. Collaborative dialogue patterns in naturalistic one-on-one tutoring. Applied Cognitive Psychology, 9:495-522.

Graesser, Arthur C., Katja Wiemer-Hastings, Peter Wiemer-Hastings, and Roger Kreuz. 1999. Autotutor: A simulation of a human tutor. Cognitive Systems Research, 1:35-51.

Houghton, G. 1986. The Production of Language in Dialogue: A Computational Model. Ph.D. thesis, University of Sussex.

Litman, Diane J. and Scott Silliman. 2004. ITSPOKE: An Intelligent Tutoring Spoken Dialogue System. In Proceedings of the Human Language Technology Conference: 4th Meeting of the North American Chapter of the Association for Computational Linguistics (HLT/NAACL) (Companion Proceedings), Boston, MA

Marineau, Johanna, Peter Wiemer-Hastings, Derek Harter, Brent Olde, Patrick Chipman, Ashish Karnavat, Victoria Pomeroy, Sonya Rajan, and Art Graesser. 2000. Classification of speech acts in tutorial dialogue. In Proceedings of the Workshop on Modeling Human Teaching Tactics and Strategies, ITS 2000, pages 65-71.

Moore, Johanna. 1993. What makes human explanations effective? In Proceedings of the $15^{\text {th }}$ Meeting of the Cognitive Science Society, pages 131-136, Hillsdale, NJ.

Porayska-Pomsta, Kaśka, Manolis Mavrikis, and Helen Pain. 2008. Diagnosing and acting on student affect: the tutor's perspective. User Modeling and User-Adapted Interaction, 18(1-2):125-173.

Tsovaltzi, Dimitra and Elena Karagjosova. 2004. A View on Dialogue Move Taxonomies for Tutorial Dialogues. In Strube, Michael and Candy Sidner, editors, Proceedings of 5th SIGdial Workshop on Discourse and Dialogue, pages 35-38, Cambridge, Massachusetts, USA. Association for Computational Linguistics.

Tsovaltzi, Dimitra, Armin Fiedler, and Helmut Horacek. 2004. A multi-dimensional taxonomy for automating hinting. In Lester, James C., Rosa Maria Vicari, and Fábio Paraguaçu, editors, Intelligent Tutoring Systems - 7th International Conference (ITS-04), number 3220 in LNCS, pages 772-781. Springer.
Wolska, M., B. Q. Vo, D. Tsovaltzi, I. KruijffKorbayova, E. Karagjosova, H. Horacek, M. Gabsdil, A. Fiedler, and C. Benzmüller. 2004. An annotated corpus of tutorial dialogs on mathematical theorem proving. In Proceedings of the Fourth International Conference on Language Resources and Evaluation (LREC-04), pages 1007-1010, Lisbon.

Zinn, Claus. 2004. Flexible dialogue management in natural-language enhanced tutoring. In Konvens 2004 Workshop on Advanced Topics in Modeling Natural Language Dialog, pages 28-35, Vienna, Austria. 


\begin{tabular}{|c|c|c|}
\hline Label & Explanation & Example \\
\hline \multicolumn{3}{|l|}{ Forward Dimension } \\
\hline Assert & Makes a claim about the world & "It holds that $P "$ \\
\hline Request & Introduces an obligation on the hearer to answer & \\
\hline Action-directive & The obligation is that an action is performed & "Please show the following" \\
\hline Info-request & Request for a piece of information & "What is the definition of...?" \\
\hline Open-option & Suggestion of future action without obligation & "You could do a proof by induction" \\
\hline \multicolumn{3}{|l|}{ Backward Dimension } \\
\hline Agreement & Acceptance or rejection of plans or propositions & \\
\hline Accept & Accepts a proposal & "Ok" \\
\hline Reject & Rejects a proposal & "That's incorrect" \\
\hline Address & Responses to requests & \\
\hline Answer & Answers a previously posed info-request & "Yes"/"No" \\
\hline Deflect & Shows inability or unwillingness to answer & "I can't answer that" \\
\hline Neutral & Addresses without answering or deflecting & "Why do you ask?" \\
\hline Information relation & Relation to an antecedent utterance & \\
\hline Understanding related & Refers to problems understanding the speaker & \\
\hline Request clarification & Asks to clarify a previous utterance & "What do you mean by X?" \\
\hline Request rephrase & Asks for a repeat/rephrase of an utterance & "Could you repeat that?" \\
\hline Signal non-understanding & Catch-all for signalling understanding problems & \\
\hline \multicolumn{3}{|l|}{ Task Dimension: Solving Task } \\
\hline Solution-step & Refers to a step to the current solution & \\
\hline Step augmentation & Adds to an existing step & "Concluded using Rule X" \\
\hline New & Contributes a new step & "Rewrite formula A to B" \\
\hline Solution-strategy & Refers to a solution strategy & \\
\hline State strategy & States a solution strategy which will be used & "I now do a case split" \\
\hline state future step & State a step that will be executed later & "I will use DeMorgan2" \\
\hline Request assistance & Ask for help with the task & \\
\hline Request concept explanation & Ask to explain a domain concept & "What does P mean?" \\
\hline Request worked example & Ask for an example to be presented & "Could you give me an example?" \\
\hline Request solution strategy & Ask what strategy to proceed with & "How should i do this task?" \\
\hline \multicolumn{3}{|l|}{ Task Dimension: Teaching Task } \\
\hline Solution-step-evaluation & References to evaluations of soln steps & \\
\hline Signal correct & Indicates the step was correct & "Correct!" \\
\hline Signal incorrect & Indicates the step was incorrect & "Wrong!" \\
\hline Hint & Give a hint towards solving the task & \\
\hline Give-away-concept & Give away a concept to help with the task & "You should use rule X" \\
\hline Request domain relevant & Requests which refer to domain concepts & \\
\hline Explain & Ask for an explanation to be given & "T: What is the defn of powerset?" \\
\hline Identify & Ask for a concept to be identified & "T: What does $\subseteq$ denote?" \\
\hline Define & Ask for a definition & "What is the definition of...?" \\
\hline \multicolumn{3}{|l|}{ Task Progress } \\
\hline Start task & Starts the solution construction process & "Please prove $P=Q "$ \\
\hline Finish task & Indicates end of the solution construction process & "I'm done", "Q.E.D" \\
\hline Restart task & Indicates solution being started again & "Start again" \\
\hline Give-up task & Abandons the current solution attempt & "I give up" \\
\hline Task solution status & References to solution progress & \\
\hline On-track & Solution construction is on track & "Am i ok?", "You're doing fine" \\
\hline Lost & Indicates speaker is lost in current solution & "I'm lost"' \\
\hline
\end{tabular}

Table 2: The full taxonomy. Each type is given along with an explanation and an example 